\title{
Special Issue on Advances in sensing and communication circuits (ICECS 2012)
}

\author{
Ángel Rodríguez-Vázquez • Jorge Fernández-Berni • \\ José M. de la Rosa
}

Published online: 12 November 2013

(c) Springer Science+Business Media New York 2013

This special issue includes 15 papers selected from some 250 presented at the 2012 International Conference on Electronics, Circuits and Systems (ICECS 2012) held December 09-12 in Seville, Spain. ICECS 2012 got 380 submissions from 51 different countries; $69.7 \%$ of the papers came from IEEE Region 8, while the rest came from the other IEEE regions. Selection of the papers for this special issue was based on: (1) the feedback of the audience; (2) the feedback of the session chairs; and (3) the scores given by the ICECS TPC members. Selected papers were invited to submit extended versions on early March 2013. All papers were peer reviewed. Papers in the issue have been organized into different section covering topics related to the general theme of sensing and communication circuits and systems. The papers are briefly introduced in the sections below.

\section{Sensors and sensory systems}

This paper by D. Jiang and A. Demosthenous deals with the merging topic of implantable neuro-prosthetic systems. It provides a review of the development in vestibular prostheses to date, and uses a specific example to demonstrate the problems in designing the implantable circuits for such systems. M. Carminati et al. addresses the topic of molecule detection by using solid-state nano-pores. The final goal is label-free high-throughput DNA sequencing.

\footnotetext{
Á. Rodríguez-Vázquez (ه) · J. Fernández-Berni .

J. M. de la Rosa

Institute of Microelectronics of Seville, University of Seville and

CNM-CSIC, Seville, Spain

e-mail: angel@imse-cnm.csic.es

J. Fernández-Berni

e-mail: berni@imse-cnm.csic.es
}

The paper presents a new two-channel current amplifier tailored for solid-state nanopore devices with integrated tunneling electrodes, analyzes different design trade-offs and show results for a lamda-DNA platform with $20 \mathrm{~nm}$ pores. A. Alfaifi et al. presents a $1 \times 1-\mathrm{mm}^{2}$ dual-axis accelerometer fabricated in a SOI process plus an in-house back-end release step. Experimental results are reported: average cross sensitivity of $\sim 0.87 \%$ with $\sim 101$ 'digital counts'/g, that is, $16.83 \mathrm{fF} / \mathrm{g}$ in both axes, $\mathrm{X}$ and $\mathrm{Y}$. A remarkable feature of this device is that rotational motion and $\mathrm{Z}$ accelerations have no impact on the $\mathrm{X}$ and $\mathrm{Y}$ readings. This is due to the particular geometry of the device. The paper by M.A. Paun et al. evaluates the temperature effects on the current-related sensitivity of Hall-Effect sensors. An analysis of the carrier concentration temperature dependence is carried out and experimental results are presented for the sensitivity and input resistance temperature dependence. The section is closed by the paper from A. Salazar et al. It presents simulation results obtained along different design stages of a biosensing platform based on Surface Plasmon Resonance (SPR) and incorporating a CMOS imager. Specifically, a numerical analysis to determine the optimal plasmon resonance conditions is reported. A numerical analysis to select the best CMOS photodiode is also described. Finally, the architecture of the corresponding CMOS imager is proposed. As a whole, this work gathers valuable information required for the successful implementation of a SPR-biosensing platform incorporating a CMOS Active Column Sensor.

\section{CMOS image and radiation sensors}

The first paper in this section, by L. Mereni et al. is devoted to circuits for passive radiometry Specifically, the paper 
address a study on the feasibility of W-band passive radiometers in nanoscale CMOS technology for imaging applications. The study takes into account some of the most relevant aspects regarding the system-on-a-chip implementation, from system to circuit and technology limitations, with emphasis on the impact of the non-idealities of the detector on the overall system performances. S.J. Carey et al. report a vision chip based on a $20 \times 64$ mixed-signal processing array. This array features elementary cells containing a photodetector, an ALU and digital and analog memory. One of the main advantages of this circuitry when arranged as a processing lattice is that the array can be configured for asynchronous propagation, enabling ultrahigh-speed operations like flood filling. Applications working at 30,000 frames per second are presented. The paper by S. Vargas-Sierra et al. reports an algorithm for acquisition of high dynamic range images. This algorithm applies the same tone mapping curve to all pixels, adapting itself dynamically to the contents of the scene. Experimental results based on a smart QCIF CMOS imager are provided. These results demonstrate the suitability of the proposed technique to compress HDR images while keeping details and contrast information for subsequent post-processing.

\section{Communication circuits and systems}

This section starts with the paper by M.P. Kennedy et al. that propose two variants of LC CMOS injection-locked frequency dividers that can operate equally well in both divide-by-2 and divide-by-3 modes. The circuits presented in this work are implemented with only one integrated inductor and designed in a $65 \mathrm{~nm}$ CMOS technology to operate within the multi-GHz frequency range. F.A. Bakar et al. present the design, implementation and experimental characterization of the analog part of an integrated receiver intended for Synthetic Aperture Radar applications. The receiver-which includes a three-parallel-signal channel RF front-end, a reconfigurable analog baseband chain with 50/160 selectable bandwidth, and a 8-bit ADC-has been fabricated in a $130 \mathrm{~nm}$ CMOS technology with $1.2-\mathrm{V}$ supply voltage, featuring a reconfigurable nominal gain of $40 / 37 \mathrm{~dB}$ and a noise figure of $11 / 13.5 \mathrm{~dB}$ for L-band and $\mathrm{C}$-band, respectively. The maximum power consumption is $650 / 800 \mathrm{~mW}$ with a $50 / 160 \mathrm{MHz}$ baseband filter, respectively. In the last paper of this section, S.M. Safavi and M. Shabany introduces a novel multiple antenna, high-resolution eigenvalue-based spectrum sensing algorithm based on the FFT of the received signal. A real-time, low-area, and low-power VLSI architecture is developed for the proposed algorithm, which is implemented in a $180 \mathrm{~nm}$ CMOS technology. This design constitutes the first eigenvalue-based detection architecture proposed to date, capable of detecting weak signals at $-10 \mathrm{~dB}$, occupying a total area of $3.4 \mathrm{~mm}^{2}$, and dissipating $78 \mathrm{~mW}$ for a 32-subchannel $40 \mathrm{MHz}$ sensing bandwidth.

\section{Design of analog front-end circuits}

The first paper of this section, by $\mathrm{T}$. Watanabe and $\mathrm{T}$. Terasawa, reports an all-digital 6- to 16-bit adaptive time A/D converter applied to high-resolution low-power sensor/RF interfaces. Minimum/maximum detectable sensitivity of $0.7 \mu \mathrm{Vrms} / 100 \mathrm{mVrms}$ is experimentally achieved in a $0.18 \mu \mathrm{m}$ CMOS process. The ultimate objective of the proposed approach is making sensor/RF ADCs more compact and scalable while easing their design and test thanks to the all-digital nature of the circuitry. B. Sedighi et al. addresses the problem of optimizing area occupation and power consumption of SAR ADCs under noise performance constraints. The paper shows that parasitic capacitances and ON-resistances of the switches play an important role in the noise performance and analyse different hybrid resistive-capacitive DACs seeking for improvements in energy efficiency. T. Rabuske et al. present two improved comparator-based binary-search ADC architectures for UWB receivers. The first ADC topology uses $2 \mathrm{~N}-1$ comparators arranged in $\mathrm{N}$ stages, and the second one uses only $\mathrm{N}$ threshold-reconfigurable comparators arranged one per stage. These two circuits are designed in a $90 \mathrm{~nm}$ CMOS technology to operate at $1.5 \mathrm{GS} / \mathrm{s}$, achieving a resolution of 4-bit and 5-bit, respectively, for a $1-\mathrm{V}$ input signal range, with a single $1-\mathrm{V}$ supply voltage. The last, but not the least, paper in the issue, by A. Buonomo and A. Lo Schiavo addresses the topic of analog modelling for equation-based design. Specifically, the paper derives closed-form expressions to predict nonlinear distortion in analog integrated circuits with application to $\mathrm{GmC}$ filters and nested Miller compensated opamps.

Acknowledgments The Guest Editors want to express their greatest appreciation to all the authors who submitted papers to the conference. Also to the authors of selected papers for the hard work they have made to incorporate all reviewer comments. Last but not least to all our reviewers for their sounded and timely reports. 


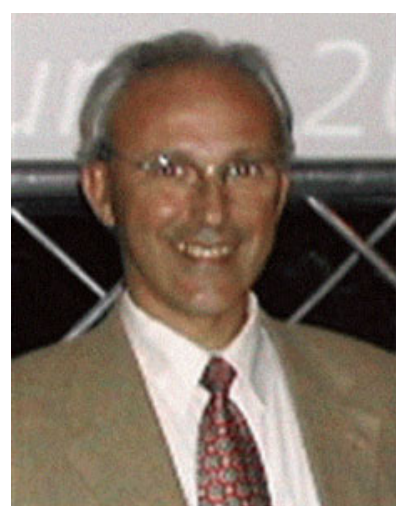

\section{Ángel Rodríguez-Vázquez}

(Ph.D., IEEE Fellow) is a Full Professor of Electronics at the University of Seville. He is also the President and the responsible for long term R\&D at AnaFocus Ltd. His research is on the design of analog and mixedsignal front-ends for sensing and communication, including smart imagers, vision chips and low-power sensory-processing microsystems. He has authored 11 books, some 43 chapters in contributed books, including original tutorials on chaotic integrated circuits, design of data converters and design of chips for vision, and some 150 journal articles in peer-review specialized publications. He has presented many invited plenary lectures at different international conferences and has received a number of international awards for his research (the IEEE Guillemin-Cauer best paper award, two Wiley's IJCTA best paper awards, two IEEE ECCTD best paper award and the IEEE ISCAS best demo-paper award). He was elected Fellow of the IEEE for his contributions to the design of chaos-based communication chips and neuro-fuzzy chips. His research work got some 5,700 quotes; he has an h-index of 39 and a i10-index of 117. He has always been looking for the balance between long term research and innovative industrial developments. He founded AnaFocus Ltd. in 2001 and served as CEO, on leave from the University, until June 2009, when the company reached maturity as a worldwide provider of smart CMOS imagers and vision systems-on-chip. He has served as Editor, Associate Editor and Guest Editor for different IEEE and non-IEEE journals, is in the committee of many international journals and conferences, and has chaired several international IEEE and SPIE conferences. He served also as VP Region 8 of the IEEE Circuits and Systems Society (2009-2012) and as Chair of the IEEE CASS Fellow Evaluation Committee (2010, 2012 and 2013).

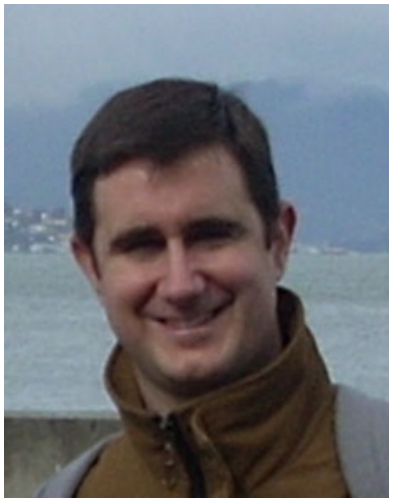

Jorge Fernández-Berni received the B.Eng. degree in Electronics and Telecommunication in September 2004 from the University of Seville, Spain. He then spent three and a half months at the Department of Instrumentation and Space Exploration of the Center for Astrobiology in Madrid, Spain, granted by the Spanish National Research Council. From January 2005 to September 2006, he was working in the telecommunication industry. In October 2006, he joined the Institute of Microelectronics of Seville as a doctoral student, receiving the
M.Sc. degree in Microelectronics in December 2008 and his Ph.D. in June 2011 with honors. He was visiting the Computer and Automation Research Institute in Budapest for a term in 2010. Since February 2011, he holds an Assistant Professorship at the Department of Electronics and Electromagnetism (University of Seville). He is the leading author of over 25 papers in refereed journals and conferences. He is also the first author of a book and a book chapter. He received the Third Prize of the Student Paper Award from the scientific committee of "IEEE CNNA 2010: Int. Workshop on Cellular Nanoscale Networks and their Applications". He is member of the IEEE CASS Technical Committee on Cellular Nanoscale Networks and Array Computing.

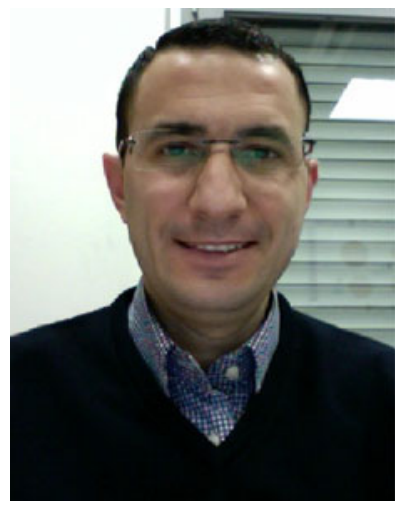

José M. de la Rosa (IEEE Senior Member) received the M.S. degree in Physics in 1993 and the Ph.D. degree in Microelectronics in 2000 , both from the University of Seville, Spain. Since 1993 he has been working at the Institute of Microelectronics of Seville, where he heads a research group on micronanoelectronic circuits and systems. He is also with the Department of Electronics and Electromagnetism of the University of Seville, where he is an Associate Professor, accredited as a Full Professor. His main research interests are in the field of analog/mixed-signal CMOS integrated circuits, especially data converters. In these topics, Dr. de la Rosa has participated in a number of Spanish and European research/industrial projects, and has co-authored some 180 international peer-reviewed publications, including journal and conference papers, books and book chapters. He is a member of the ASPTC of the IEEE CAS Society. He serves as Associate Editor for IEEE Transactions on Circuits and Systems I: Regular Papers. He is also a member of the Steering Committee of MWSCAS and he serves in the technical committees of diverse IEEE conferences, including ISCAS, MWSCAS, ICECS, LASCAS, VLSI-SoC and DATE, being TPC co-chair of MWSCAS' 12 and ICECS' 12. 